\title{
Cellular electrophysiologic and mechanical evidence of superior vascular protection in pulmonary microcirculation by Perfadex compared with Celsior
}

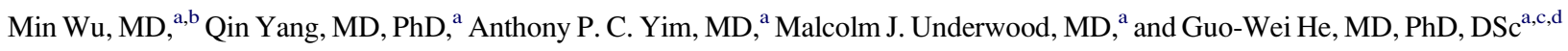

\begin{abstract}
Objective: Pulmonary endothelial function is critical in posttransplant lung performance. Hyperkalemic organ preservation solutions alter vascular endothelial function through the non-nitric oxide and non-prostacyclin pathway, but the most frequently used lung preservation solutions, Perfadex (Vitrolife Sweden, Kungsbacka, Sweden) $\left(\mathrm{K}^{+} 6 \mathrm{mmol} / \mathrm{L}\right)$ and Celsior (IMTIX SangStat Company, Lyon, France) $\left(\mathrm{K}^{+} 15 \mathrm{mmol} / \mathrm{L}\right)$, have not been evaluated on pulmonary endothelial protection. We compared the non-nitric oxide and non-prostacyclin-mediated endothelial function in porcine pulmonary microarteries of lung preserved by Perfadex or Celsior solution at $4{ }^{\circ} \mathrm{C}$ for 4 hours.
\end{abstract}

\begin{abstract}
Methods: The non-nitric oxide and non-prostacyclin-mediated endothelial function was determined by measuring the membrane potential in a single pulmonary smooth muscle cell (group II, $\mathrm{n}=6$ ) and bradykinin-induced relaxation (group I, $\mathrm{n}=8$ ) in pulmonary microarteries preserved in Krebs (a, control), Perfadex (b), or Celsior (c), with inhibitors of nitric oxide and prostacyclin.
\end{abstract}

Results: Membrane potential hyperpolarization decreased in IIc $(4.5 \pm 0.2 \mathrm{mV}, P<.05)$ but was preserved $(P>$ $.05)$ in IIa $(6.6 \pm 0.1 \mathrm{mV})$ and $\mathrm{IIb}(6.3 \pm 0.3 \mathrm{mV})$. Resting membrane potential was depolarized in IIc $(-42.8 \pm 1.3$ $\mathrm{mV})$ compared with IIa $(-58.7 \pm 0.6 \mathrm{mV})$ and $\mathrm{IIb}(-56.7 \pm 0.9 \mathrm{mV})(P<.05)$. Hyperpolarization-associated relaxation $(37.3 \% \pm 7.2 \%$ vs $59.7 \% \pm 7.7 \%)$ and sensitivity $\left(\mathrm{EC}_{50}\right)(-7.29 \pm 0.13$ vs $-7.75 \pm 0.06 \log \mathrm{M})$ to bradykinin significantly $(P<.05)$ decreased in Ic but not in Ia and Ib.

Conclusion: This in vitro study simulating clinical conditions demonstrates that Perfadex preserves endotheliumdependent smooth muscle relaxation and hyperpolarization better than Celsior solution in regard to the electrophysiologic and mechanical properties observed at cellular and vascular levels. This study provides a new method at the level of basic science to evaluate the solutions for heart/lung preservation.

To obtain the best results in lung transplantation, the preservation of donor lung is evaluated. Since the early reports on safe lung preservation for 12 to 24 hours with Perfadex (Vitrolife Sweden, Kungsbacka, Sweden), ${ }^{1,2}$ this solution has gained wide use in lung transplantation surgery. Compared with Euro-Collins (EC) solution, a reduction of perioperative mortality and morbidity in clinical trials has demonstrated that Perfadex provides superior maintenance of graft function after pulmonary transplantation and results in better long-term survival. ${ }^{3,4}$ A recent study ${ }^{5}$ suggested that Perfa-

From the Department of Surgery, The Chinese University of Hong Kong, ${ }^{a}$ Hong Kong; Guangdong Provincial People's Hospital, ${ }^{\text {b }}$ Guangzhou, China; Medical College, Nankai University and TEDA International Cardiovascular Hospital, ${ }^{\mathrm{c}}$ Tianjin, China; Providence Heart and Vascular Institute, Albert Starr Academic Center, Department of Surgery, Oregon Health and Science University, ${ }^{\mathrm{d}}$ Portland, Oregon.

Supported by grants from the Research Grants Council of the Hong Kong Special Administrative Region (Project No. CUHK 4651/07M) and the Direct Grants 2041164, 4450171, 2041305, 2041384, and 2041388 of the Chinese University of Hong Kong, Hong Kong, SAR China and the Providence St. Vincent Medical Foundation, Portland, OR

Received for publication Dec 28, 2007; revisions received May 29, 2008; accepted for publication Aug 28, 2008.

Address for reprints: Guo-Wei He, MD, PhD, DSc, Department of Surgery, The Chinese University of Hong Kong, 5A, Block B, Prince of Wales Hospital, Shatin,

N.T., Hong Kong SAR, China (E-mail: gwhe@cuhk.edu.hk).

J Thorac Cardiovasc Surg 2009;137:492-8

$0022-5223 / \$ 36.00$

Copyright (c) 2009 by The American Association for Thoracic Surgery doi:10.1016/j.jtcvs.2008.08.047 dex prevented moderate-to-severe posttransplant pulmonary graft dysfunction; therefore, the use of Perfadex instead of EC (or Papworth) should reduce pulmonary graft dysfunction in lung transplantation. Perfadex is also reported to be useful in the preservation of lungs from elderly donors. ${ }^{6}$

On the other hand, Celsior (IMTIX SangStat Company, Lyon, France) solution, a high-sodium, low-potassium, extracellular-type solution (Table 1), was formulated specifically for heart preservation and designed to prevent cellswelling, oxygen-derived free radical injury and contracture by enhancement of energy production and limitation of calcium overload. ${ }^{7,8}$ This solution was then developed as one of the alternative solutions for pulmonary preservation ${ }^{9,10}$ and was demonstrated to improve the function of porcine pulmonary grafts stored for 24 hours. ${ }^{11}$

In lung preservation, the protection of endothelial function has become an important issue because endothelium plays an important role in regulating the vascular tone of pulmonary circulation. It is well known that nitric oxide (NO), prostacyclin $\left(\mathrm{PGI}_{2}\right)$, and a non-NO and non-PGI 2 (NNONPG) pathway, also referred to as endothelium-derived hyperpolarizing factor (EDHF) released by vascular endothelium, contribute to endothelium-dependent relaxation. ${ }^{12} \mathrm{We}$ have demonstrated that hyperkalemia alters the coronary endothelial function through the NNONPG pathway ${ }^{13,14}$ and that the mechanism is the reduced hyperpolarization of the smooth 


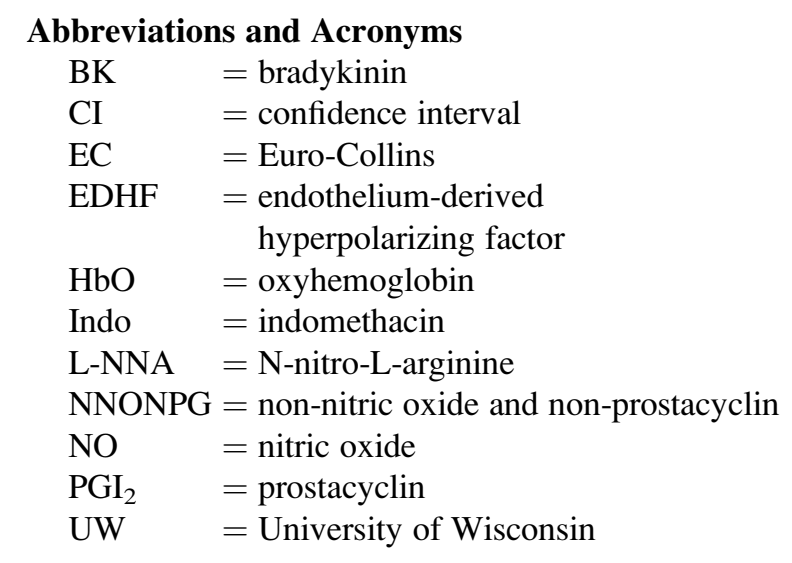

muscle cell caused by the depolarizing effect of high potassium concentration. ${ }^{13-16}$ We demonstrated that clinically used hyperkalemic solutions, such as St Thomas Hospital solution (containing $\left.16-20 \mathrm{mmol} / \mathrm{L} \mathrm{K} \mathrm{K}^{+}\right),{ }^{17}$ University of Wisconsin (UW) solution (containing $\left.125 \mathrm{mmol} / \mathrm{L} \mathrm{K}^{+}\right){ }^{18}$ and the relatively low- $\mathrm{K}^{+}$solution, Celsior (containing 15 $\left.\mathrm{mmol} / \mathrm{L} \mathrm{K}^{+}\right),{ }^{19}$ alter the EDHF-mediated endothelial function in the porcine coronary circulation. In particular, we reported the harmful effect of hyperkalemic lung preservation solutions, including EC (containing $115 \mathrm{mmol} / \mathrm{L} \mathrm{K}^{+}$) and UW solutions, on EDHF-mediated hyperpolarization and relaxation. ${ }^{20,21}$

Although it has been reported ${ }^{21}$ that extracellular-type solutions (ie, Perfadex [containing $6 \mathrm{mmol} / \mathrm{L} \mathrm{K}^{\dagger}$ ] and Celsior) are associated with better lung preservation than intracellular-type solutions in clinical transplantation, the comparative effect between Perfadex and Celsior solutions remains controversial. ${ }^{22-24}$

The present study was therefore designed to evaluate the effects of Perfadex and Celsior solutions on the NNONPG (EDHF)-mediated relaxation and associated cellular hyperpolarization under conditions similar to those in the clinical setting.

\section{MATERIALS AND METHODS}

All experiments were performed in accordance with institutional guidelines. This investigation conformed to the "Guide for the Care and Use of Laboratory Animals," published by the US National Institutes of Health (Publication No. 85-23, revised 1996).

\section{Vessel Preparation}

Fresh porcine lungs collected from a local slaughterhouse were placed in a container filled with cold Krebs solution $\left(4^{\circ} \mathrm{C}\right)$ and immediately transferred to the laboratory. The transportation time was less than 1 hour. On receipt of the lung, small (micro) pulmonary arteries (usually the third branches of the left or right apical lobe, diameter 300-600 $\mu \mathrm{m}$ ) were carefully dissected and removed under a microscope within 1 hour. The vessels were cleaned of fat and connective tissue and cut into cylindric rings $2 \mathrm{~mm}$ in length under a microscope. The ring was guided with a suitable length of stainless steel wires ( $40 \mu \mathrm{M}$ in diameter) through its lumen. The wire was
TABLE 1. Composition of Perfadex and Celsior solutions compared with the physiologic solution (Krebs)

\begin{tabular}{|c|c|c|c|}
\hline Composition & Krebs & $\begin{array}{c}\text { Celsior } \\
\text { (IMTIX SangStat } \\
\text { Company, Lyon, } \\
\text { France) }\end{array}$ & $\begin{array}{c}\text { Perfadex } \\
\text { (Vitrolife Sweden, } \\
\text { Kungsbacka, } \\
\text { Sweden) }\end{array}$ \\
\hline $\mathrm{Na}^{+}(\mathrm{mmol} / \mathrm{L})$ & 143.4 & 100 & 138 \\
\hline $\mathrm{K}^{+}(\mathrm{mmol} / \mathrm{L})$ & 5.9 & 15 & 6 \\
\hline $\mathrm{Ca}^{2+}(\mathrm{mmol} / \mathrm{L})$ & 2.5 & 0.26 & 0.27 \\
\hline $\mathrm{Mg}^{2+}(\mathrm{mmol} / \mathrm{L})$ & 1.2 & 13 & 0.8 \\
\hline $\mathrm{Cl}^{-}(\mathrm{mmol} / \mathrm{L})$ & 128.7 & 41.5 & 142 \\
\hline $\mathrm{HCO}_{3}^{-}(\mathrm{mmol} / \mathrm{L})$ & 25 & - & - \\
\hline $\mathrm{SO}_{4}^{2-}(\mathrm{mmol} / \mathrm{L})$ & 1.2 & 0 & 0.8 \\
\hline $\mathrm{H}_{2} \mathrm{PO}_{4}^{-}(\mathrm{mmol} / \mathrm{L})$ & 1.2 & 0 & 0.8 \\
\hline $\mathrm{HPO}_{4}{ }^{2-}(\mathrm{mmol} / \mathrm{L})$ & - & - & - \\
\hline Glucose (mmol/L) & 11.1 & 0 & 5 \\
\hline Lactobionic acid (g/L) & - & 80 & - \\
\hline THAM (mmol/L) & - & - & 1 \\
\hline Dextran $(g / L)$ & - & - & 50 \\
\hline Histidine (mmol/L) & - & 30 & - \\
\hline Mannitol (mmol/L) & - & 60 & - \\
\hline Glutathione & - & 3 & - \\
\hline Glutamate & - & 20 & - \\
\hline Osmolarity (mosmol/kg) & 319 & 360 & 325 \\
\hline
\end{tabular}

fixed tightly on a jaw in a 2-channel myograph (model 500A; JP Trading, Aarhus, Denmark), and another wire was passed lightly through the vascular lumen and then anchored the other jaw of the same chamber. These 2 wires were attached to a force transducer or micrometer. An adjustable micrometer can be used to pull the jaws apart, stretching the artery between the 2 parallel wires. A calibrated force transducer was used to measure the force with the output shown on a computer screen, and the graphs were printed in a printer. Data were digitized and stored in the computer. The Krebs solution (Table 1) was aerated with a gas mixture of $95 \%$ oxygen and $5 \%$ carbon dioxide at $37^{\circ} \mathrm{C}$ during preparation.

\section{Normalization}

After the ring was mounted in the myograph and equilibrated for $30 \mathrm{~min}$ utes, a previously described method ${ }^{25}$ was used to normalize the ring under a condition simulating the transmural pressure encountered in vivo in the pulmonary microcirculation. Briefly, the arterial rings were progressively stretched until the passive transmural pressure reached $40 \mathrm{~mm} \mathrm{Hg}$, and the pressure was immediately released. The computerized program determines the length-tension exponential curve for each ring and gives the internal circumference and diameter at a pressure of $40 \mathrm{~mm} \mathrm{Hg}$. The internal circumference was then set to a value estimated to be equivalent to $90 \%$ of the circumference at a passive transmural pressure of $40 \mathrm{~mm} \mathrm{Hg}$ throughout the experiment. The vessels were then released to the normalized value. This degree of passive tension at this setting is the passive or resting situation in the absence of constricting tone and was maintained throughout the experiments. In the myograph model $500 \mathrm{~A}$, the normalization procedure was performed automatically.

\section{Cellular Electrophysiologic Study}

The myograph was mounted within a Faraday metal-screened cage. A conventional glass microelectrode, filled with $3 \mathrm{~mol} / \mathrm{L} \mathrm{KCl}$ (tip resistance, 40-80 $\mathrm{M} \Omega$ ), was advanced using a pipette holder mounted on a 3-dimensional vernier-type hydraulic micromanipulator and inserted into a single smooth muscle cell from the adventitial surface of the rings. The electrical signals were amplified by means of a battery-operated, low-noise, wideband microelectrode amplifier electrometer (Electro 705; World Precision 
Instruments Inc, Sarasota, Fla). The output signals were monitored continuously on a dual-trace oscilloscope (model 2120 B; World Precision Instruments Inc) and simultaneously recorded by a computer with the installed PicoScope program (Pico Technology Limited, Hardwick, UK). The following criteria were used to assess the validity of a successful impalement: (1) a sudden negative shift in voltage, followed by (2) a stable negative voltage for more than 2 minutes, and (3) an instantaneous return to the previous voltage level on dislodgement of the microelectrode, as previously reported. ${ }^{19,26}$

\section{Experimental Protocols}

All rings were equilibrated at $37^{\circ} \mathrm{C}$ and aerated with $95 \%$ oxygen and $5 \%$ carbon dioxide for 30 minutes before and after normalization. The following protocols were used.

\section{Isometric Force Study (Group I; $\mathbf{n}=8$ in Each Subgroup): Bradykinin-induced, EDHF-mediated \\ Relaxation}

In the relaxation studies, N-nitro-L-arginine (L-NNA) $(300 \mu \mathrm{M})$, an NO synthase inhibitor, oxyhemoglobin ( $\mathrm{HbO}, 20 \mu \mathrm{M})$, an NO scavenger, and indomethacin (Indo, $7 \mu \mathrm{M}$ ), a cyclooxygenase inhibitor, were added to the chamber for 30 minutes. A thromboxane $\mathrm{A}_{2}$ mimetic $\mathrm{U}_{46619}(-8 \mathrm{log}$ $M)$ was then added to contract the rings. When the contraction reached a stable plateau (usually 15 minutes), cumulative concentration-relaxation curves to bradykinin (BK) $(-10$ to $-6.5 \log \mathrm{M})$ were established.

The arteries were then stored in Krebs (group Ia, control), Perfadex (group Ib), or Celsior (group Ic) solution at $4^{\circ} \mathrm{C}$ for 4 hours, followed by washout with Krebs within 45 minutes at $37^{\circ} \mathrm{C}$. The NNONPG-mediated relaxation to $\mathrm{BK}$ was induced again. In pilot experiments under normalization, after a washout procedure with Krebs solution and equilibrium for a certain period, the repeated BK-induced, NNONPG (EDHF)-mediated relaxation in the $\mathrm{U}_{46619}$-precontraction remained unchanged.

\section{Cellular Electrophysiologic Study (Group II; $\mathbf{n}=6$ in Each Subgroup): NNONPG-mediated Cellular Hyperpolarization and Associated Relaxation}

The small pulmonary arterial rings were mounted in the myograph and then normalized to the physiologic pressure. The micrometer parameters were recorded, and the pressure was then released. The rings were then incubated in Krebs (group IIa, control), Perfadex (group IIb), or Celsior (group IIc) solution at $4^{\circ} \mathrm{C}$ for 4 hours, similar to the above protocol. The rings were then set at the previous micrometer parameters to be under physiologic pressure. In the presence of Indo, L-NNA, and $\mathrm{HbO}$ in the myograph chamber, the resting membrane potential and resting force were simultaneously recorded. When Perfadex or Celsior solution was replaced with Krebs within 30 minutes at $37^{\circ} \mathrm{C}$, the NNONPG-mediated cellular hyperpolarization (in a single smooth muscle cell) and associated relaxation induced by BK (-7.0 $\log \mathrm{M})$ were recorded. During this period, the cellular membrane potential and the force were continuously measured.

\section{Data Analysis}

Two-way analysis of variance was used for comparison in isometric force studies. Paired Student $t$ test was used to calculate the difference of self-control comparison on the NNONPG-mediated hyperpolarization. One-way analysis of variance or unpaired Student $t$ test was used to calculate the difference between groups.

\section{Drugs}

The drugs used and their sources were as follows: BK, L-NNA, Indo, and hemoglobin were from Sigma Chemical Co (St Louis, Mo). Solutions of L-NNA (dissolved in distilled water) and Indo (dissolved in ethanol) were stored at $4^{\circ} \mathrm{C}$. Commercial bovine hemoglobin was dissolved in $0.9 \%$ saline solution to make up a 3-mL stock solution. The stock solution was subsequently reduced to $\mathrm{HbO}$ by the addition of a small amount $(<0.3 \mathrm{~g})$ of sodium dithionite. Excessive sodium dithionite was extracted by running the solution through an Econo-Pac 10DG column (Bio-Rad, Hercules, Calif) equilibrated with $0.9 \%$ saline solution. The $\mathrm{HbO}$ solutions were frozen in aliquots at $-20^{\circ} \mathrm{C}$ and stored for up to 14 days. The Celsior solution was purchased from IMTIX SangStat Company (Lyon, France). Perfadex solution was donated by Vitrolife Sweden (Kungsbacka, Sweden).

\section{RESULTS \\ Relaxation Study: NNONPG-mediated Relaxation (Group I)}

Resting force. No significant differences were detected before and after cold storage in Perfadex or Celsior solutions in each group regarding the resting force (group Ia: $2.5 \pm 0.2$ $\mathrm{mN}$ vs $2.4 \pm 0.3 \mathrm{mN}, P=.10,95 \% \mathrm{CI},-0.029-0.246$; group Ib: $2.7 \pm 0.2 \mathrm{mN}$ vs $2.7 \pm 0.3 \mathrm{mN}, P=.95,95 \%$ CI, $-0.131-0.123$; group Ic: $2.6 \pm 0.5 \mathrm{mN}$ vs $2.6 \pm 0.3$ $\mathrm{mN}, P=.97,95 \% \mathrm{CI},-0.482-0.467)$.

$\mathbf{U}_{46619}$-induced precontraction. The concentration of $\mathrm{U}_{46619}$ varied from $-8 \log \mathrm{M}$ to $-7.7 \log \mathrm{M}$ to maintain a similar stable contraction force before and after exposure to Perfadex or Celsior solution in each group (group Ia $6.1 \pm 0.5$ $\mathrm{mN}$ vs $6.4 \pm 0.7 \mathrm{mN}, P=.56,95 \% \mathrm{CI},-1.077$ to 0.632 ; group $\mathrm{Ib}: 7.5 \pm 0.6 \mathrm{mN}$ vs $8.0 \pm 0.7 \mathrm{mN}, P=.14,95 \%$ CI, -1.316 to 0.234 ; group Ic: $6.8 \pm 0.8 \mathrm{mN}$ vs $7.3 \pm 0.8$ $\mathrm{mN}, P=.2,95 \% \mathrm{CI},-1.465$ to 0.335$)$.

\section{NNONPG (EDHF)-mediated Relaxation}

Group Ia (control). No significant differences in the maximal NNONPG (EDHF)-mediated relaxation by BK were detected before and after cold storage in Krebs solution $(66.2 \% \pm 6.1 \%$ vs $61.8 \% \pm 2.6 \%, P=.36 ; 95 \%$ CI, -6.32 to 15.1 ; Figure $1, A$ ).

Group Ib. After cold storage in Perfadex solution, no significant differences were detected in the maximal NNONPG (EDHF)-mediated relaxation $(63.3 \% \pm 5.5 \%$ vs $56.8 \% \pm$ $3.9 \%, P=.17,95 \% \mathrm{CI},-3.9 \%$ to $16.9 \%$; Figure $1, B)$, with no significant change of the $\mathrm{EC}_{50}(-7.86 \pm 0.09$ vs $-7.81 \pm 0.1 \log \mathrm{M}, P=.76,95 \% \mathrm{CI},-0.45$ to $0.34 \log \mathrm{M})$.

Group Ic. In contrast, after incubation in Celsior solution, the maximal NNONPG (EDHF)-mediated relaxation to BK decreased from $59.7 \% \pm 7.7 \%$ to $37.3 \% \pm 7.2 \%$, $P<.01 ; 95 \%$ CI, 11.5-33.4, (Figure 1, $C$ ). In addition, the $\mathrm{EC}_{50}$ significantly increased from $-7.75 \pm 0.06$ to $-7.29 \pm 0.13(P<.05,95 \%$ CI, 0.08-0.84 $\log \mathrm{M})$.

Further, although there were no significant differences in the relaxation before incubation between group $\mathrm{Ib}$ and group Ic $(63.3 \% \pm 5.5 \%$ vs $59.7 \% \pm 7.7 \%, 95 \% \mathrm{CI},-16.6 \%$ to $23.9 \%, P>.05)$, the NNONPG-mediated relaxation was significantly higher after incubation in Perfadex (group Ib: $56.8 \% \pm 3.9 \%$ ) than after incubation in Celsior (group Ic: $37.3 \% \pm 7.2 \% ; P<.05,95 \%$ CI, $2 \%-37.1 \%)$. This 


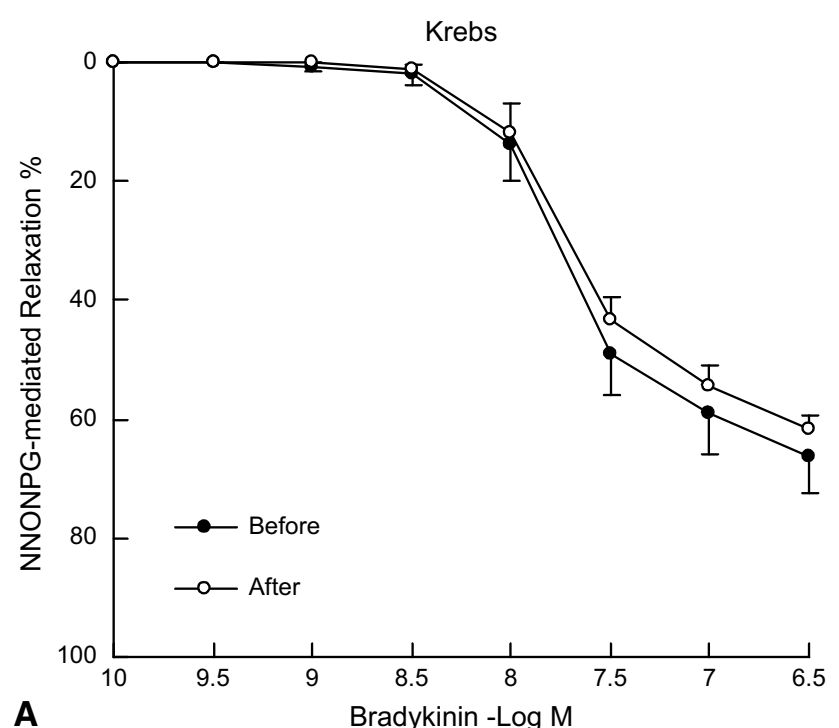

A
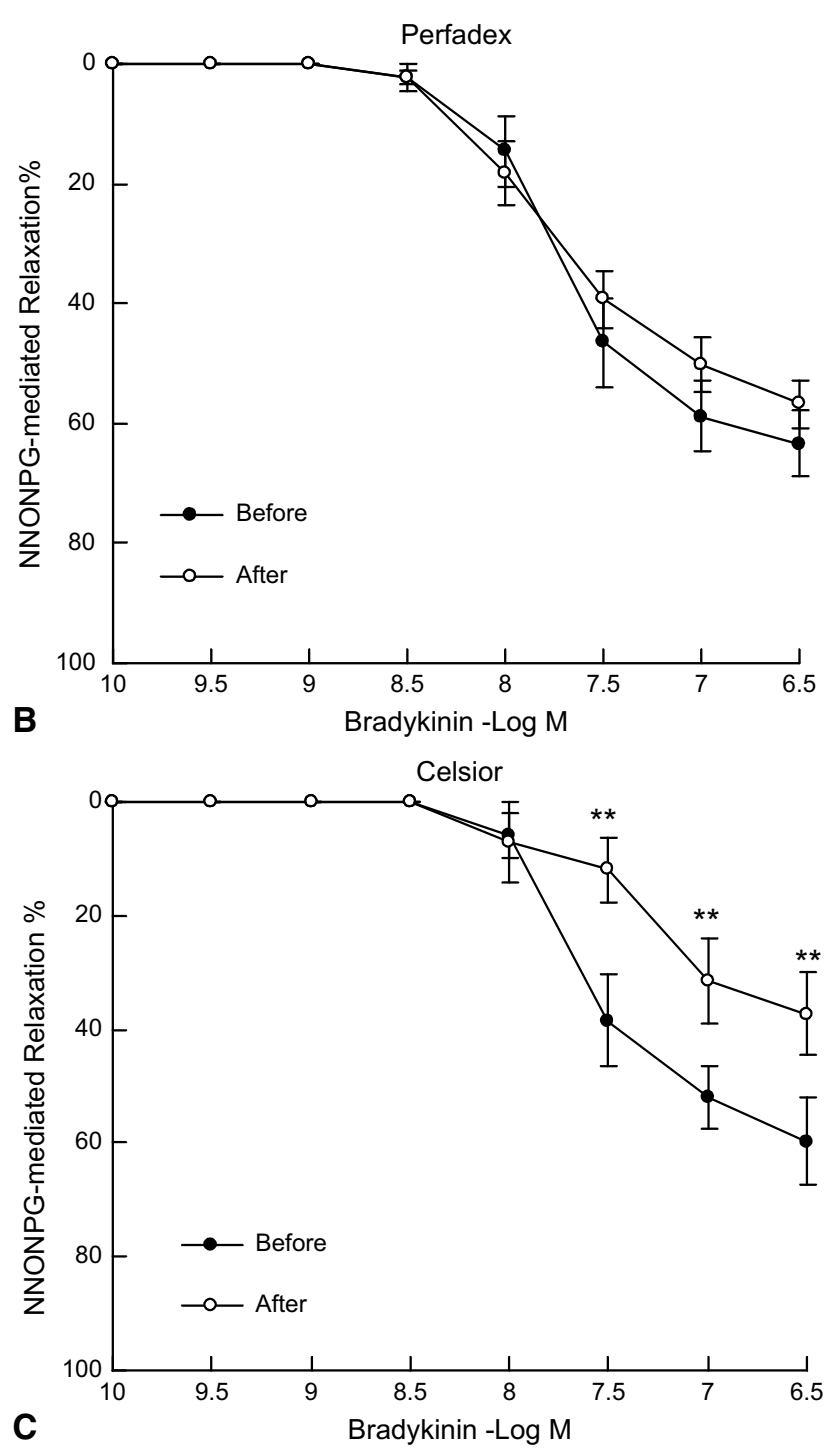

difference also suggests a protective effect of Perfadex solution on the NNONPG-mediated relaxation compared with Celsior solution.

\section{Electrophysiologic Study (Group II): The Resting Cellular Membrane Potential of Single Smooth Muscle Cell}

In comparison with the vessel incubated in Perfadex (group IIb: $-56.7 \pm 0.9 \mathrm{mV}, \mathrm{n}=6$ ) or Krebs solution (group IIa: $-58.7 \pm 0.6 \mathrm{mV}, \mathrm{n}=6$ ), the resting membrane potential of a single smooth muscle cell was depolarized after incubation in Celsior solution even within 30 minutes after washout with Krebs solution (group IIc: $-42.8 \pm 1.3 \mathrm{mV}, P<.01$ compared with group IIa or group IIb) (Figures 2 and 3).

\section{NNONPG (EDHF)-mediated Cellular Hyperpolarization}

The NNONPG-mediated cellular hyperpolarization was significantly reduced after incubation in Celsior solution (group IIc: $4.5 \pm 0.2 \mathrm{mV}, 95 \% \mathrm{CI}, 2.0-4.4 \mathrm{mV}$ ) compared with Krebs $(6.6 \pm 0.1 \mathrm{mV}, P<.01)$ or Perfadex solution (6.3 $\pm 0.3 \mathrm{mV}, P<.01$ ) (Figure 4). No significant differences were detected on EDHF-mediated cellular hyperpolarization between Perfadex and Krebs solution $(P=.36)$.

\section{Cellular Hyperpolarization-associated Relaxation}

During the measurement of the cellular membrane potential, the associated relaxation was simultaneously recorded. Because hyperpolarization and relaxation were induced from the resting status without precontraction tone, hyperpolarization-associated relaxation was in a small range $(0.32 \pm$ $0.02 \mathrm{mN}$ in group IIa, Krebs solution). The relaxation after incubation in Celsior solution (group IIc: $0.2 \pm 0.02 \mathrm{mN}$, $P<.01,95 \% \mathrm{CI},-0.16$ to $-0.07 \mathrm{mN}$ ) was significantly reduced compared with Perfadex incubation (group IIb: $0.3 \pm$ $0.02 \mathrm{mN}, P<.01)$. In contrast, no significant differences in hyperpolarization-associated relaxation were detected after incubation between Perfadex and Krebs $(P>.05)$ (Figure 4).

\section{DISCUSSION}

This in vitro study demonstrates that the clinically used lung preservation solution Perfadex is superior to Celsior solution in terms of the vascular preservation of the pulmonary resistance artery regarding the NNONPG pathway. This

FIGURE 1. Concentration-relaxation curves for BK (-10 to $-6.5 \log \mathrm{M})$ in the $\mathrm{U}_{46619}(10 \mathrm{nM})$-precontracted pulmonary microarteries in the presence of Indo $(7 \mu \mathrm{M})$, L-NNA $(300 \mu \mathrm{M})$, and $\mathrm{HbO}(20 \mu \mathrm{M})$ before (control; solid circles) and after (open circles) cold storage in Krebs (A; group Ia), Perfadex (Vitrolife Sweden, Kungsbacka, Sweden) (B; group Ib), or Celsior (IMTIX SangStat Company, Lyon, France) (C; group Ic) solution at $4{ }^{\circ} \mathrm{C}$ for 4 hours. Data are shown as mean \pm standard error of the mean. $* P<.05$. $* * P<.01$ compared with the control group $(\mathrm{n}=8$, paired $t$ test). NNONPG, Non-nitric oxide and non-prostacyclin. 

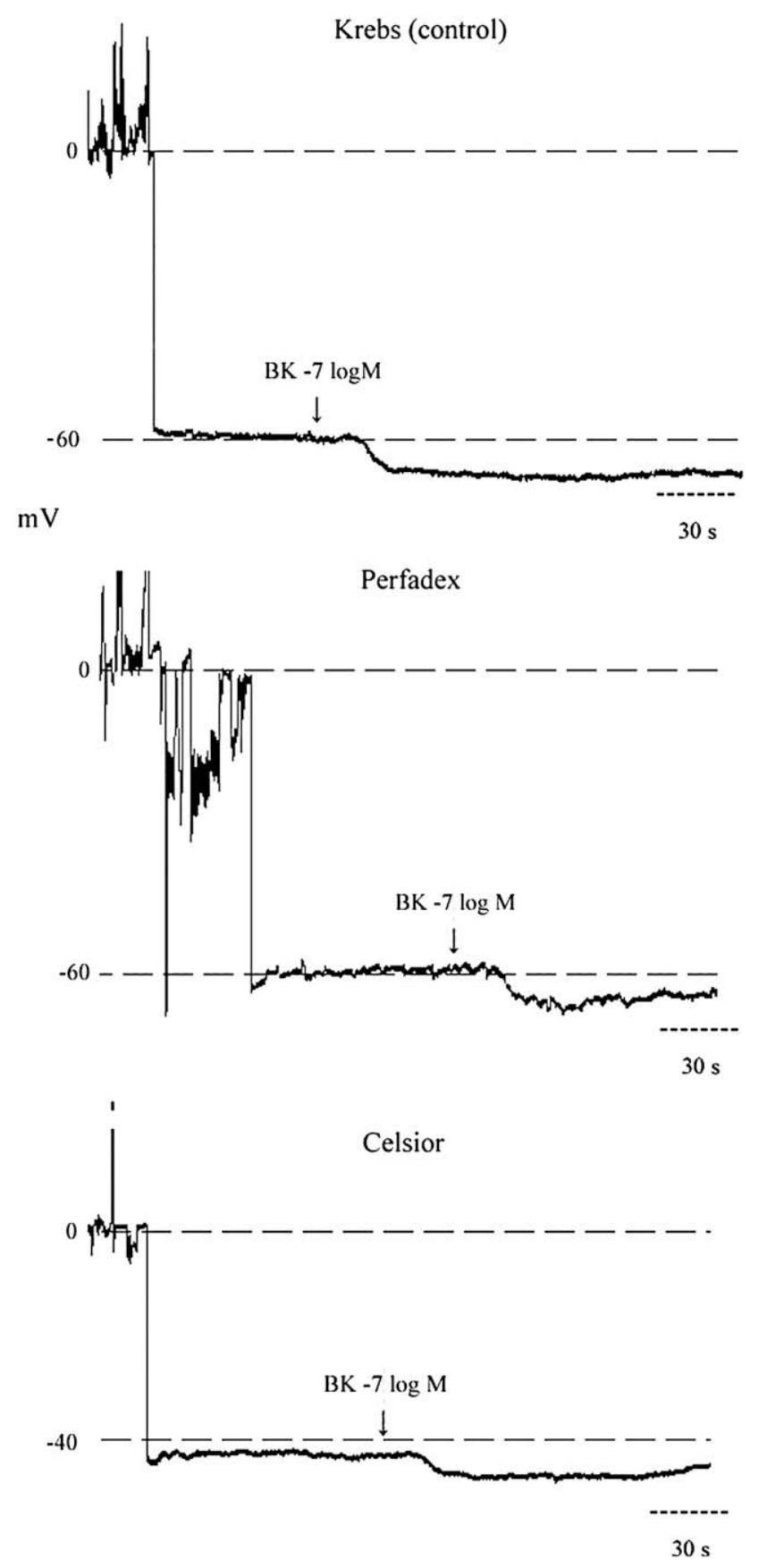

FIGURE 2. Original tracings of NNONPG(EDHF)-mediated hyperpolarization induced by $\mathrm{BK}(-7 \log \mathrm{M})$ in porcine pulmonary arteries in the presence of Indo $(7 \mu \mathrm{M})$, L-NNA $(300 \mu \mathrm{M})$, and $\mathrm{HbO}(20 \mu \mathrm{M})$ after incubation in Krebs (control), Perfadex, or Celsior solution at $4^{\circ} \mathrm{C}$ for 4 hours. BK, Bradykinin.

finding may explain the superior clinical results in using Perfadex solution.

Celsior is well known as a relatively new preservation solution for heart transplantation. In recent years, Celsior has also been shown to improve the protection of pulmonary grafts. As reported, ${ }^{9}$ compared with the low-potassium EC

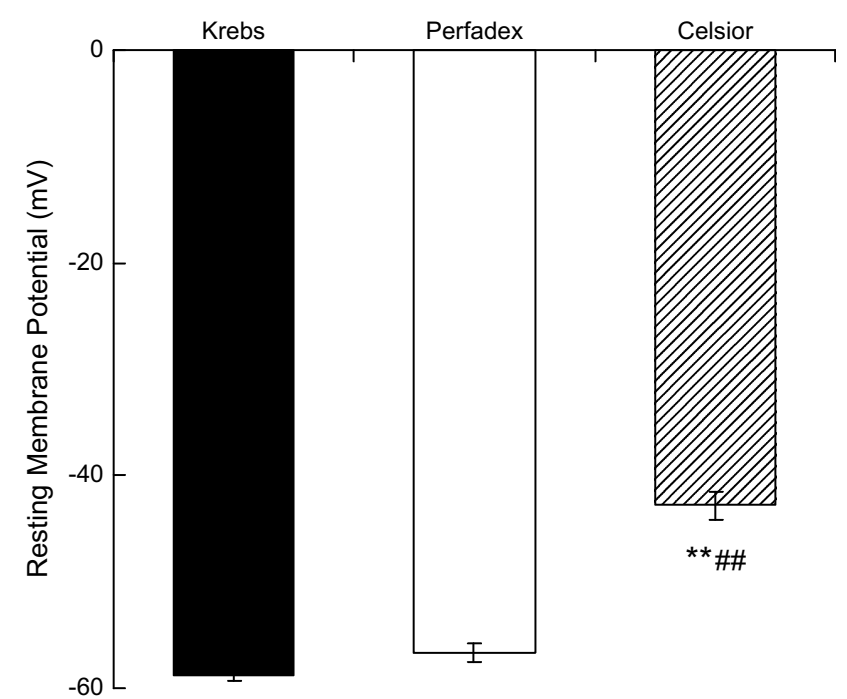

FIGURE 3. The resting membrane potential from small pulmonary arteries after exposure to Krebs (control, group IIa), Perfadex (group IIb), or Celsior (group IIc) solution at $4^{\circ} \mathrm{C}$ for 4 hours followed by washout with Krebs solution within 30 minutes in the presence of Indo $(7 \mu \mathrm{M})$, L-NNA $(300 \mu \mathrm{M})$, and $\mathrm{HbO}(20 \mu \mathrm{M})$. ** or \#\#P<.01 compared with control (Krebs**) or Perfadex (\#\#) group. Data are shown as mean \pm standard error of the mean.

solution (containing $40 \mathrm{mmol} / \mathrm{L}$ of potassium), Celsior solution provides a significantly increased oxygenation ability, lower pulmonary vascular resistance, and decreased wet/ dry ratio during the lung preservation. Studies have reported that Celsior solution improved the endothelial viability and proliferative capability of endothelial cells in cultured human greater saphenous vein, ${ }^{27}$ and that endothelin and inducible NO synthase gene expression were significantly higher after cold storage in Celsior solution compared with UW solution. ${ }^{28}$ On the other hand, Celsior solution contains $15 \mathrm{mmol} / \mathrm{L} \mathrm{K}^{+}$that is still hyperkalemic, although the $\mathrm{K}^{+}$ concentration is relatively lower compared with UW or other hyperkalemic solutions. We have demonstrated that in porcine resistance coronary arteries, Celsior solution ${ }^{19}$ impairs EDHF-mediated endothelial function.

The present study clearly shows that Celsior solution depolarizes the resting cellular membrane potential of the pulmonary smooth muscle cell (Figures 2 and 3 ) and reduces the NNONPG (EDHF)-mediated hyperpolarization and associated relaxation (Figure 4) after cold storage at $4^{\circ} \mathrm{C}$ for 4 hours, and that the effect exists at least for 30 minutes after washout with warm Krebs.

In contrast, Perfadex, as an almost normokalemic solution containing only $6 \mathrm{mmol} / \mathrm{L} \mathrm{K}^{+}$, has been reported to provide sufficient lung preservation for 27 hours of cold ischemia and significant functional and histologic improvement with retrograde perfusion. ${ }^{29}$ However, the effect of Perfadex solution on cellular membrane hyperpolarization related to pulmonary endothelial function has not been investigated. We hypothesized that because of the normokalemic 

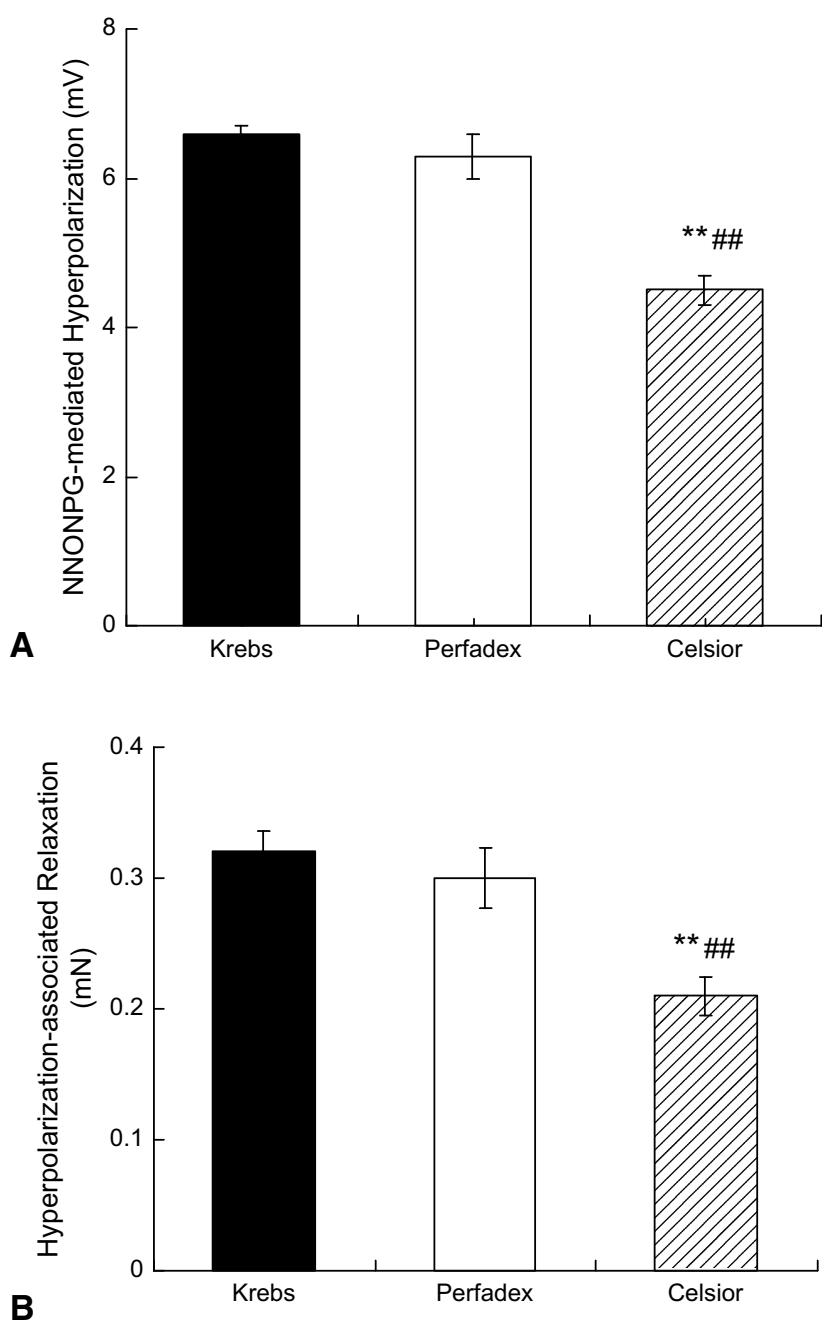

FIGURE 4. The NNONPG (EDHF)-mediated hyperpolarization (A) and associated relaxation (B) from small pulmonary arteries induced by BK significantly after exposure to Perfadex solution (group IIb) or Celsior (group IIc) solution at $4^{\circ} \mathrm{C}$ for 4 hours in the presence of Indo $(7 \mu \mathrm{M})$, L-NNA (300 $\mu \mathrm{M})$, and $\mathrm{HbO}(20 \mu \mathrm{M})$, followed by washout with Krebs solution within 30 minutes. Note that both hyperpolarization (A) and associated relaxation (B) remain after exposure to Perfadex solution but decreased after exposure to Celsior solution. ** or \#\#P<.01 compared with control (group IIa, Krebs**) or Perfadex (\#\#) group. Data are shown as mean \pm standard error of the mean. NNONPG, Non-nitric oxide and non-prostacyclin.

components in Perfadex solution, it may maintain the cellular membrane potential of the pulmonary smooth muscle cell at the normal level, and this is why the NNONPG-mediated endothelial function is preserved. The present study supports this hypothesis and demonstrates that after cold storage at $4^{\circ} \mathrm{C}$ for 4 hours, unlike Celsior solution that depolarizes the membrane potential, Perfadex does not alter the resting membrane potential of the pulmonary smooth muscle cell and NNONPG (EDHF)-mediated endothelial function, including relaxation and the associated cellular membrane hyperpolarization.

As discussed above, on the basis of the present and our previous studies, ${ }^{13-21}$ the differences in the NNONPG
(EDHF)-mediated hyperpolarization and relaxation after incubation with Perfadex and Celsior solutions are most likely due to the difference in the potassium concentration in these solutions.

Ischemia-reperfusion injury predominately injures the alveolar capillary endothelial cells, resulting in loss of barrier function and alveolar edema accumulation. The present study does not provide information on the endothelium at the blood-air barrier because the study model concerns the endothelium-smooth muscle interaction at the resistance artery level in the lung, that is, the present study investigates the preservation of the pulmonary endothelium at the vascular level and not the alveolar level. The experimental model in the present study is not suitable to investigate the alveolar endothelium. However, a recent morphologic study demonstrates that Perfadex effectively prevents intra-alveolar, septal, and peribronchovascular edema formation, as well as injury of the blood-air barrier during ischemia-reperfusion. Celsior was not effective in preserving the lung from morphologic ischemia-reperfusion injury. ${ }^{30}$

On the other hand, an intact vasculature is an essential part of normal lung function. A damaged vasculature, particularly at the level of resistance vessel, would change the vascular tone. For example, the mechanical and electronic change of the endothelium-smooth muscle interaction at the resistance vessel level seen in the present study-with damaged NNONPGH function-would increase the vascular tone and arterial resistance, which may affect the perfusion flow to the transplanted lung after transplantation. ${ }^{30}$ In lung transplantation, it has been demonstrated that the release of NO is diminished $^{31,32}$ because of the damage to the vascular endothelium. The present study, from a new angle-the NNONPG pathway-demonstrates the effect of the preservation solutions. The NNONPG pathway (often referred to as EDHF) is a backup mechanism for NO. ${ }^{12}$ Therefore, the present study demonstrates that in addition to NO, the second pathway to modulate the pulmonary vascular toneNNONPG (inhibited by smooth muscle depolarization) - is damaged by Celsior but preserved by Perfadex solution. The clinical relevance is therefore obvious that Perfadex may protect the vascular endothelium in the lung to better modulate the perfusion flow to the transplanted lung.

Table 1 shows a comparison of the compositions of these 2 preservation solutions with the normal physiologic solution (Krebs), which has simple physiologic compositions. The composition of Perfadex is closer to Krebs in the concentration of $\mathrm{Na}^{+}, \mathrm{K}^{+}, \mathrm{Mg}^{2+}, \mathrm{Cl}^{-}, \mathrm{SO}_{4}{ }^{2-}$, and $\mathrm{H}_{2} \mathrm{PO}_{4}{ }^{-}$. The major difference is its content of dextran $(50 \mathrm{~g} / \mathrm{L})$. The addition of the large molecule dextran is believed to be important in lung preservation. Dextran probably has a crucial role in the preservation of vascular endothelial/smooth muscle cells and pulmonary parenchymal cells. Krebs solution is not used for heart or lung preservation most likely because physiologic solutions do not sufficiently preserve parenchymal 
cells in the heart (cardiac myocytes) and lung; therefore, specific solutions are designed for this purpose.

\section{Limitations}

The present study is an in vitro experimental investigation at the tissue (vascular) and cellular level. The observed effect on the NNONPG function of the endothelium-dependent smooth muscle relaxation and hyperpolarization of the pulmonary arteries in vivo remains to be further defined. In addition, the lung tissue was first preserved in cold Krebs solution for transportation and then subjected to exposure to the preservation solutions, although the establishment of the control group (Krebs) may eliminate this influence. The study was performed in porcine pulmonary arteries, and differences between this vasculature and human vasculature should be kept in mind.

\section{Conclusions}

Both Perfadex and Celsior solutions have been used clinically for lung transplantation and shown to decrease ischemia-reperfusion injury of the donor lung and to improve lung function in lung transplantation. The present study has provided evidence that in regard to the protection of pulmonary endothelium-dependent smooth muscle relaxation and hyperpolarization, Perfadex is superior to Celsior solution at both the cellular and vascular levels. Further investigations are warranted to study these effects in patients.

This study was fully supported by grants from the Research Grants Council of the Hong Kong Special Administrative Region (Project No. CUHK 4651/07M) and the Direct Grant 2041164, 4450171, 2041305, 2041384, 2041388 of The Chinese University of Hong Kong, Hong Kong, SAR China and the Providence St Vincent Medical Foundation, Portland, Ore.

\section{References}

1. Steen S, Sjoberg T, Massa G, Ericsson L, Lindberg L. Safe pulmonary preservation for 12 hours with low-potassium-dextran solution. Ann Thorac Surg. 1993; 55:434-40.

2. Steen S, Kimblad PO, Sjoberg T, Lindberg L, Ingemansson R, Massa G. Safe lung preservation for twenty-four hours with Perfadex. Ann Thorac Surg. 1994;57:450-7.

3. Muller C, Furst H, Reichenspurner H, Briegel J, Groh J, Reichart B. Lung procurement by low-potassium dextran and the effect on preservation injury. Munich Lung Transplant Group. Transplantation. 1999;68:1139-43.

4. Struber M, Wilhelmi M, Harringer W, Niedermeyer J, Anssar M, Kunsebeck A, et al. Flush perfusion with low potassium dextran solution improves early graft function in clinical lung transplantation. Eur J Cardiothorac Surg. 2001;19:190-4.

5. Oto T, Griffiths AP, Rosenfeldt F, Levvey BJ, Williams TJ, Snell GI. Early outcomes comparing Perfadex, Euro-Collins, and Papworth solutions in lung transplantation. Ann Thorac Surg. 2006;82:1842-8.

6. Fischer S, Gohrbandt B, Struckmeier P, Niedermeyer J, Simon A, Hagl C, et al. Lung transplantation with lungs from donors fifty years of age and older. J Thorac Cardiovasc Surg. 2005;129:919-25.

7. Warnecke G, Schulze B, Haverich A, Klima U. Celsior solution provides superior post-ischemic right ventricular function as compared with UW solution in a porcine heart transplantation model. J Heart Lung Transplant. 2002;21:586-9.

8. Menasche P, Termignon JL, Pradier F, Grousset C, Mouas C, Alberici G, et al. Experimental evaluation of Celsior, a new heart preservation solution. Eur J Cardiothorac Surg. 1994;8:207-13.
9. Wittwer T, Wahlers T, Cornelius JF, Elki S, Haverich A. Celsior solution for improvement of currently used clinical standards of lung preservation in an ex vivo rat model. Eur J Cardiothorac Surg. 1999;15:667-71.

10. Large SR. Pulmonary preservation with LPD and Celsior solution in porcine lung transplantation after $24 \mathrm{~h}$ of cold ischemia. Eur J Cardiothorac Surg. 2004;26:157.

11. Sommer SP, Gohrbandt B, Fischer S, Hohlfeld JM, Warnecke G, Avsar M, et al. Glutathione improves the function of porcine pulmonary grafts stored for twentyfour hours in low-potassium dextran solution. J Thorac Cardiovasc Surg. 2005; 130:864-9.

12. Ge ZD, Zhang XH, Fung PCW, He GW. Endothelium-dependent hyperpolarization and relaxation resistance to NG-nitro-1-arginine and indomethacin in coronary circulation. Cardiacovasc Res. 2000;46:547-56.

13. He GW, Yang CQ, Graier WF, Yang JA. Hyperkalemia alters EDHF-mediated hyperpolarization and relaxation in coronary arteries. Am J Physiol. 1996;271:H760-7.

14. He GW, Yang CQ. Hyperkalemia alters endothelium-dependent relaxation through non-nitric oxide and noncyclooxygenase pathway: a mechanism for coronary dysfunction due to cardioplegia. Ann Thorac Surg. 1996;61:1394-9.

15. He GW, Yang CQ, Yang JA. Depolarizing cardiac arrest and endothelium-derived hyperpolarizing factor-mediated hyperpolarization and relaxation in coronary arteries: the effect and mechanism. J Thorac Cardiovasc Surg. 1997;113:932-41.

16. Yang Q, He GW. Effect of cardioplegic and organ preservation solutions and their components on coronary endothelium-derived relaxing factors. Ann Thorac Surg. 2005;80:757-67.

17. Ge ZD, He GW. Altered EDHF-mediated endothelial function in coronary microarteries by St. Thomas' Hospital solution. J Thorac Cardiovasc Surg. 1999;118: 173-80.

18. Ge ZD, He GW. Comparison of University of Wisconsin and St. Thomas' Hospital solutions on endothelium-derived hyperpolarizing factor-mediated function in coronary micro-arteries. Transplantation. 2000;70:22-31.

19. Wu M, Yang Q, Dong YY, Yim APC, He GW. Cellular electrophysiological and mechanical effects of Celsior solution on endothelial function in resistance coronary arteries. Transplantation. 2005;80:1765-72.

20. Zou W, Yang Q, Yim APC, He GW. Impaired EDHF-mediated relaxation in porcine pulmonary micro-arteries after cold storage with Euro-Collins and University of Wisconsin solutions. J Thorac Cardiovasc Surg. 2003;126:208-15.

21. Zhang RZ, Yang Q, Yim AP, He GW. Alteration of cellular electrophysiologic properties in porcine pulmonary microcirculation after preservation with University of Wisconsin and Euro-Collins solutions. Ann Thorac Surg. 2004;77:1944-50.

22. Thabut G, Vinatier I, Brugiere O, Leseche G, Loirat P, Bisson A. Influence of preservation solution on early graft failure in clinical lung transplantation. Am J Respir Crit Care Med. 2001;164:1204-8.

23. Wittwer T, Wahlers T, Fehrenbach A, Elki S, Haverich A. Improvement of pulmonary preservation with Celsior and Perfadex: impact of storage time on early post-ischemic lung function. J Heart Lung Transplant. 1999;18:1198-201.

24. Fehrenbach A, Pufe T, Wittwer T, Nagib R, Dreyer N, Pech T, et al. Reduced vascular endothelial growth factor correlates with alveolar epithelial damage after experimental ischemia and reperfusion. J Heart Lung Transplant. 2003;22:967-78.

25. He GW, Acuff TE, Ryan WH, Yang CQ, Douthit MB, Bowman RT, et al. Inhibitory effects of calcium antagonists on alpha-adrenoceptor-mediated contraction in the human internal mammary artery. Br J Clin Pharmacol. 1994;37:173-9.

26. He GW, Liu ZG. Comparison of NO release and endothelium-derived hyperpolarizing factor-mediated hyperpolarization between human radial and internal mammary arteries. Circulation. 2001;104:I-344-9.

27. Alamanni F, Parolari A, Visigalli R, Bussolati O, Rubini P, Sala R, et al. Endothelial cell injury induced by preservation solutions: a confocal microscopy study. Ann Thorac Surg. 2002;73:1606-15.

28. Wildhirt SM, Weis M, Schulze C, Conrad N, Rieder G, Enders G. Effects of Celsior and University of Wisconsin preservation solutions on hemodynamics and endothelial function after cardiac transplantation in humans: a single-center, prospective, randomized trial. Transpl Int. 2000;13(suppl 1):S203-11.

29. Wittwer T, Franke UF, Fehrenbach A, Ochs M, Sandhaus T, Schuette A, et al. Experimental lung transplantation: impact of preservation solution and route of delivery. J Heart Lung Transplant. 2005;24:1081-90.

30. Mühlfeld C, Müller K, Pallesen LP, Sandhaus T, Madershahian N, Richter J, et al. Impact of preservation solution on the extent of blood-air barrier damage and edema formation in experimental lung transplantation. Anat Rec (Hoboken). 2007;290:491-500.

31. Bhabra MS, Hopkinson DN, Shaw TE, Hooper TL. Attenuation of lung graft reperfusion injury by a nitric oxide donor. J Thorac Cardiovasc Surg. 1997;113:327-33.

32. Vainikka T, Heikkilä L, Kukkonen S, Toivonen HJ. L-Arginine in lung graft preservation and reperfusion. J Heart Lung Transplant. 2001;20:559-67. 